glucocorticoid induction regimen, or relapse severity) had a significant differential effect on the primary outcome. By 24 months after entry, 20 months after randomization, $11 / 85(13 \%)$ patients in the RTX group had experienced a relapse compared to $32 / 85$ (38\%) patients in the AZA group. $19 / 85(22 \%)$ patients in the RTX group and $31 / 85$ (36\%) patients in the AZA group experienced at least one severe adverse event (SAE). 25/85 (29\%) and 42/85 (49\%) patients in the RTX group developed hypogammaglobulinaemia ( $\operatorname{lgG}<5 \mathrm{~g} / \mathrm{l})$ and non-severe infections respectively, compared to $21 / 85$ (25\%) and $41 / 85$ (48\%) in the AZA group.

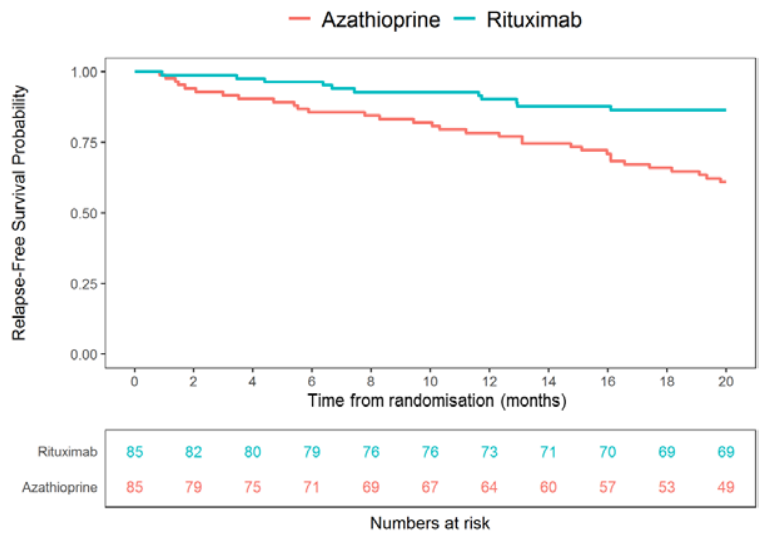

Figure 1. Relapse-free survival in RITAZAREM trial: rituximab versus azathioprine

Conclusion: In the RITAZAREM trial, following induction of remission with RTX, RTX was superior to AZA for preventing disease relapse in patients with AAV with a prior history of relapse. There were no new major safety signals for use of these medications in this population.

Disclosure of Interests: Rona Smith Grant/research support from: Roche, David Jayne Grant/research support from: ChemoCentryx, GSK, Roche/Genentech, Sanofi-Genzyme, Consultant of: Astra-Zeneca, ChemoCentryx, GSK, InflaRx, Takeda, Insmed, Chugai, Boehringer-Ingelheim, Peter A. Merkel Grant/research support from: AstraZeneca, Bristol-Myers Squibb, Boeringher-Ingelheim, Celgene, ChemoCentryx, Genentech/Roche. Genzyme/Sanofi, GlaxoSmithKline, InflaRx, Kypha, TerumoBCT., Consultant of: AbbVie, AstraZeneca, Biogen, Bristol-Myers Squibb, Boeringher-Ingelheim, Celgene, ChemoCentryx, Genentech/Roche, Genzyme/Sanofi, GlaxoSmithKline, InflaRx, Insmed, Jannsen, Sparrow, Kiniksa. DOI: 10.1136/annrheumdis-2020-eular.2717

\section{OP0027 \\ TIME TO FLARE AND GLUCOCORTICOID EXPOSURE IN PATIENTS WITH NEW-ONSET VERSUS RELAPSING GIANT CELL ARTERITIS TREATED WITH TOCILIZUMAB OR PLACEBO PLUS PREDNISONE TAPERING: 3-YEAR RESULTS FROM A RANDOMIZED CONTROLLED PHASE 3 TRIAL}

J. H. Stone $^{1}$, H. Spotswood ${ }^{2}$, S. Unizony ${ }^{1}$, M. Aringer ${ }^{3}$, D. Blockmans ${ }^{4}$, E. Brouwer ${ }^{5}$, M. C. Cid $^{6}$, B. Dasgupta ${ }^{7}$, J. Rech ${ }^{8}$, C. Salvarani ${ }^{9}$, R. Spiera ${ }^{10}$, M. Bao ${ }^{11}$.

${ }^{1}$ Massachusetts General Hospital Rheumatology Unit, Harvard Medical School, Boston, United States of America; ${ }^{2}$ Roche Products Ltd, Welwyn Garden City, United Kingdom; ${ }^{3}$ University Medical Center and Faculty of Medicine, TU Dresden, Dresden, Germany; ${ }^{4}$ Department of General Internal Medicine, University Hospitals Gasthuisberg, Leuven, Belgium; ${ }^{5}$ Department of Rheumatology and Clinical Immunology, University of Groningen, Groningen, Netherlands; ${ }^{6}$ Department of Autoimmune Diseases, Hospital Clínic, University of Barcelona, Institut d'Investigacions Biomèdiques August Pi i Sunyer, Barcelona, Spain; ${ }^{7}$ Southend University Hospital, NHS Foundation Trust, Westcliff-on-Sea, United Kingdom; ${ }^{8}$ Friedrich-Alexander-University Erlangen-Nürnberg, Department of Internal Medicine 3-Rheumatology and Immunology, Universitätsklinikum Erlangen, Erlangen, Germany; ${ }^{9}$ Division of Rheumatology, Arcispedale Santa Maria NuovaIRCCS, Reggio Emilia, Italy; ${ }^{10}$ Hospital for Special Surgery, Cornell, United States of America; ${ }^{11}$ Genentech, South San Francisco, United States of America

Background: In part 1 of the 52-week, double-blind GiACTA trial, tocilizumab (TCZ) every week $(\mathrm{QW})$ or every other week (Q2W) + prednisone tapering reduced the risk for flare versus placebo $(\mathrm{PBO})+26$-week prednisone tapering among patients with new-onset giant cell arteritis (GCA) at baseline. Among patients with relapsing GCA, TCZ QW but not Q2W + prednisone reduced the risk for flare versus both $\mathrm{PBO}$ groups, and there was separation in the time to flare between the TCZ QW and Q2W groups. ${ }^{1}$

Objectives: To report time to first flare and potential cumulative glucocorticoid (GC) sparing over 3 years of the GiACTA trial (part 1+2-year open-label part 2) among patients with new-onset or relapsing GCA.
Methods: At the end of part 1, patients entered open-label part 2, in which GCA therapy (including initiation/termination of open-label TCZ and/or GCs) was given at the investigator's discretion according to disease status. Time to first GCA flare during the 3-year study period was assessed using Kaplan-Meier analysis for patients in the intention-to-treat population according to disease onset status at baseline (new-onset/relapsing) based on their originally assigned treatment groups: TCZ QW TCZ Q2W, or pooled PBO (PBO+26-week and PBO+52-week prednisone taper) Results: Among patients randomly assigned in part 1, 47 of $100(47 \%)$ in the TCZ QW group, 26 of 49 (53\%) in the TCZ Q2W group, and 46 of $101(46 \%)$ in the pooled PBO group had new-onset GCA at baseline; the rest had relapsing GCA. Median time to first flare over 3 years was longer for patients assigned to TCZ treatment in part 1 than for patients assigned to PBO; Kaplan-Meier analysis showed a clear separation between the TCZ QW and the pooled PBO groups over 3 years for patients with new-onset and relapsing GCA (Figure 1A). Separation between the TCZ QW and TCZ Q2W groups was also observed over 3 years in patients with new-onset and relapsing GCA, although this was more evident in patients with relapsing GCA (Figure 1B). Higher proportions of patients in the TCZ QW group (new-onset, 49\%; relapsing, 47\%) than the pooled PBO group (new-onset, $28 \%$; relapsing, $31 \%$ ) and the TCZ Q2W group (new-onset, 27\%; relapsing, 35\%) remained flare-free during their entire treatment period. Cumulative prednisone dose over 3 years was lower for patients originally assigned to TCZ QW versus those originally assigned to PBO for patients with new-onset GCA and those with relapsing GCA at baseline (Figure 2). Conclusion: In this 3-year analysis of GiACTA parts 1 and 2, time to first flare favored TCZ QW over TCZ Q2W in patients with new-onset and relapsing GCA. TCZ QW delayed time to first flare and resulted in lower cumulative GC exposure compared with PBO in patients with new-onset and relapsing GCA, supporting TCZ QW dosing in patients with GCA regardless of disease onset.

References:

[1] Stone JH et al. N Engl J Med 2017;377:317-28.
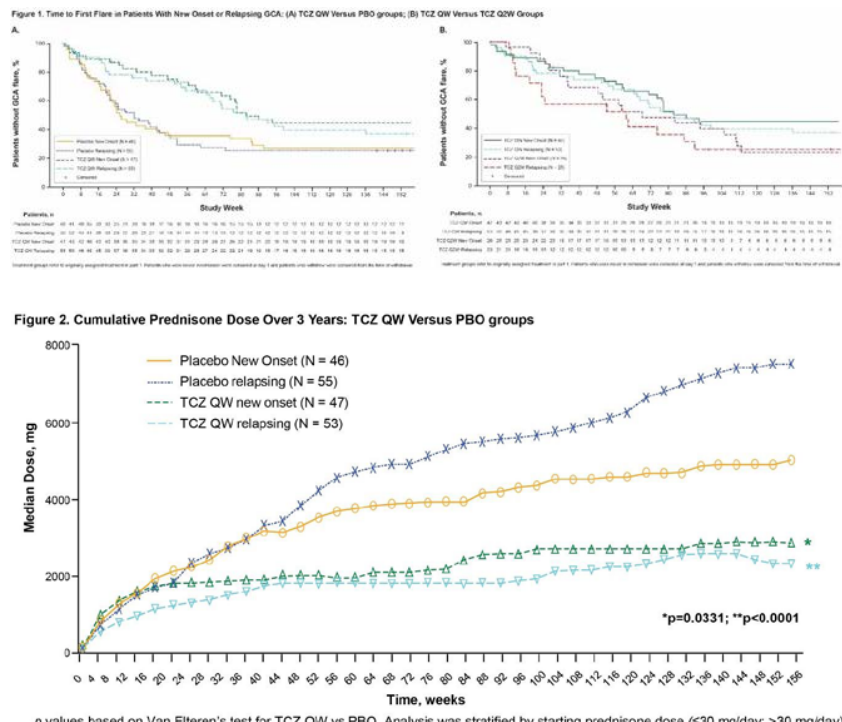

Disclosure of Interests: John H. Stone Grant/research support from: Roche, Consultant of: Roche, Helen Spotswood Shareholder of: Roche Products Ltd, Employee of: Roche Products Ltd, Sebastian Unizony Grant/research support from: Genentech, Inc., Martin Aringer Consultant of: Boehringer Ingelheim, Roche, Speakers bureau: Boehringer Ingelheim, Roche, Daniel Blockmans Consultant of: yes, Speakers bureau: yes, Elisabeth Brouwer Consultant of: Roche (consultancy fee 2017 and 2018 paid to the UMCG), Speakers bureau: Roche (2017 and 2018 paid to the UMCG), Maria C. Cid Speakers bureau: Roche, Bhaskar Dasgupta Grant/research support from: Roche Consultant of: Roche, Sanofi, GSK, BMS, AbbVie, Speakers bureau: Roche, Jürgen Rech Consultant of: BMS, Celgene, Novartis, Roche, Chugai, Speakers bureau: AbbVie, Biogen, BMS, Celgene, MSD, Novartis, Roche, Chugai, Pfizer, Lilly, Carlo Salvarani: None declared, Robert Spiera Grant/research support from: Roche-Genetech, GSK, Boehringer Ingelheim, Chemocentryx, Corbus, Forbius, Sanofi, Inflarx, Consultant of: Roche-Genetech, GSK, CSL Behring, Sanofi, Janssen, Chemocentryx Forbius, Mistubishi Tanabe, Min Bao Shareholder of: Roche, Employee of: Genentech DOI: 10.1136/annrheumdis-2020-eular. 1538

\begin{tabular}{|l|l}
\hline OP0028 & EFFICACY OF APREMILAST FOR THE PAIN OF ORAL \\
ULCERS ASSOCIATED WITH ACTIVE BEHÇET'S \\
SYNDROME: 12-WEEK RESULTS FROM THE \\
RANDOMIZED, PHASE III RELIEF STUDY
\end{tabular}

G. Hatemi ${ }^{1}$, A. Mahr ${ }^{2}$, M. Takeno ${ }^{3}$, D. $\mathrm{Kim}^{4}$, M. Melikoglu' ${ }^{1}$, S. Cheng ${ }^{5}$, S. Mccue ${ }^{5}$, S. Richter ${ }^{5}$, M. Brunori ${ }^{5}$, M. Paris ${ }^{5}$, M. Chen ${ }^{5}$, Y. Yazici ${ }^{6}{ }^{1}{ }^{1}$ stanbul 
University-Cerrahpaşa, Cerrahpaşa Medical School and Behçet's Disease Research Center, Istanbul, Turkey; ${ }^{2}$ Cantonal Hospital St. Gallen, St. Gallen, Switzerland; ${ }^{3}$ Nippon Medical School, Graduate School of Medicine, Tokyo, Japan; ${ }^{4}$ Yonsei University College of Medicine and Severance Hospital, Seoul, Korea, Rep. of (South Korea); ${ }^{5}$ Amgen Inc., Thousand Oaks, United States of America; ${ }^{6}$ New York University School of Medicine, New York, United States of America

Background: Oral ulcers (OU) associated with Behçet's syndrome are often painful, may interfere with the ability to eat and can negatively affect quality of life. ${ }^{1,2}$ Apremilast (APR), an oral phosphodiesterase 4 inhibitor, demonstrated efficacy in the treatment of OU associated with Behçet's syndrome in a phase III, multicenter, randomized, double-blind, placebo (PBO)-controlled study (RELIEF; BCT-002). ${ }^{3}$

Objectives: To describe the efficacy of APR treatment in improving OU pain associated with Behçet's syndrome in RELIEF.

Methods: Patients were randomized (1:1) to APR $30 \mathrm{mg}$ twice daily (APR 30 $\mathrm{BID}$ ) or PBO twice daily for a 12-week PBO-controlled phase, followed by a 52 -week active treatment extension. Eligible patients were $\geq 18$ years of age and had active Behçet's syndrome with $\geq 3$ OU at randomization or $\geq 2$ OU at screening and randomization and without active major organ involvement. Clinical improvement in OU was evaluated by the area under the curve for the number of $\mathrm{OU}$ through Week 12 ( $\mathrm{AUC}_{\mathrm{Wk0}-12}$; primary efficacy endpoint) and by assessments of OU number. Patient-reported OU pain was evaluated by the $100-\mathrm{mm}$ visual analogue scale (VAS). The statistical tests were 2-sided $(a=0.05)$. The proportions of patients achieving the minimal clinically important difference (MCID) and higher rates of improvement, defined as $\geq 10$ $\mathrm{mm},{ }^{4} \geq 30$-mm (3-fold MCID), $\geq 50$-mm (5-fold MCID) improvements in OU pain VAS scores, respectively, were analyzed through Week 12. An ANCOVA model was used to analyze the primary endpoint and assessments of OU number and OU pain (VAS). The proportion of patients achieving improvement in OU pain VAS scores at Week 12 were summarized descriptively.

Results: A total of 207 patients were randomized and received $\geq 1$ dose of study medication (APR: $n=104$; PBO: $n=103$ ). At baseline, the mean (SD) number of OU was 4.2 (3.7) in the APR 30 BID group and 3.9 (2.7) in the PBO group, and the mean (SD) OU pain VAS scores were 61.2 (27.6) and 60.8 (26.9), respectively. At Week 12, significantly greater improvements were observed with APR 30 BID vs. PBO in AUC $_{\text {Wk0-12 }}$ (least-squares [LS] mean [SE]: 129.5 [15.9] vs. 222.1 [15.9]; $P<0.0001$ ), number of OU (LS mean [SE]: 1.1 [0.2] vs. 2.0 [0.3]; $P=0.0003$ ) and OU pain VAS scores (LS mean [SE] change from baseline: -40.7 [3.3] vs. -15.9 [3.3]; $P<0.0001)$. The proportion of patients who achieved the MCID of $\geq 10-\mathrm{mm}$ improvement in OU pain VAS scores at Week 12 was greater with APR 30 BID vs. PBO; this pattern was also observed for the higher 3- and 5-fold improvements in MCID (Figure 1). Greater proportions of APR 30 BID vs. PBO patients achieved $\geq 10-\mathrm{mm}$ and $\geq 30$-mm improvements in OU pain VAS scores over 12 weeks. Notably, greater achievement of $\geq 50-\mathrm{mm}$ improvement in OU pain VAS scores was observed with APR 30 BID vs. PBO as early as Week 1 and maintained up to Week 12 (Figure 2).

Conclusion: For patients with active Behçet's syndrome, APR 30 BID provided significantly greater improvements vs. $\mathrm{PBO}$ in $\mathrm{OU}$ number and $\mathrm{OU}$ pain at Week 12, including the greater proportion of patients achieving MCID and 3- and 5-fold MCID of OU pain in the APR 30 BID group vs. the PBO group. These results indicate a clinically meaningful treatment effect of APR 30 BID on the OU associated with Behçet's syndrome.

References:

[1] Kokturk A. Patholog Res Int. 2012;2012:690390.

[2] Hatemi G, et al. Ann Rheum Dis. 2008;67:1656-1662.

[3] Hatemi G, et al. N Engl J Med. 2019;381:1918-1928. 4. Dworkin RH, et al. J Pain. 2008;9:105-121.

Figure 1. Proportion of Patients Achieving Improvements in OU Pain VAS at Week 12

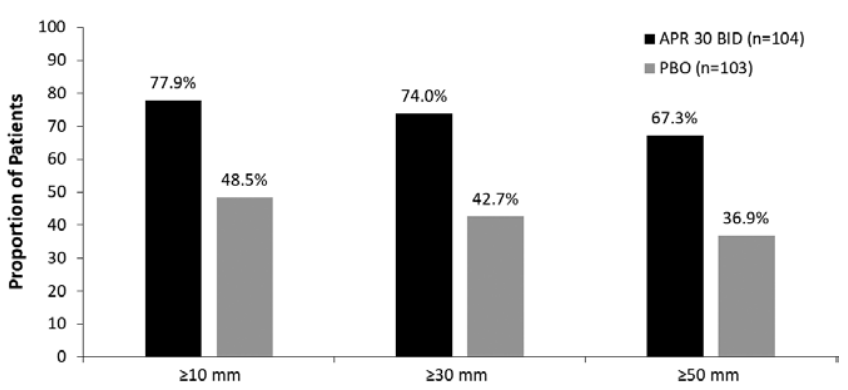

Improvement From Baseline in OU Pain VAS (0-100 mm)
Figure 2. Proportion of Patients Achieving $\geq 50-\mathrm{mm}$ Improvements in OU Pain VAS Over 12 Weeks

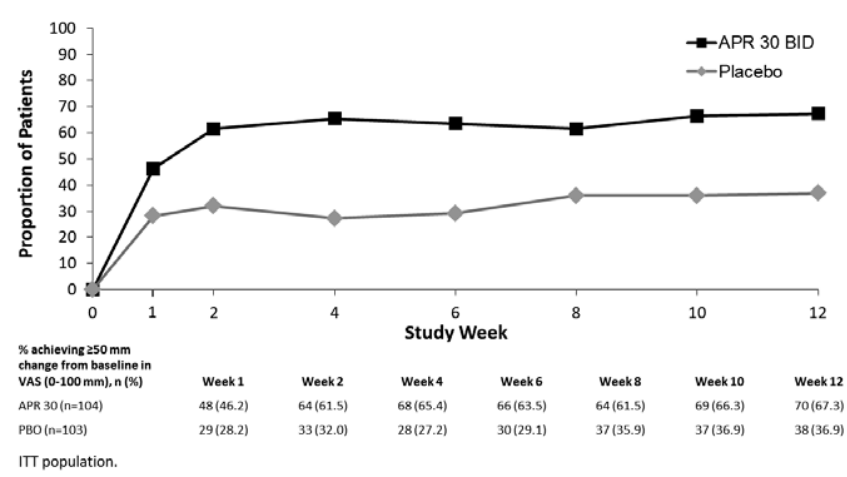

Disclosure of Interests: Gulen Hatemi Grant/research support from: BMS, Celgene Corporation, Silk Road Therapeutics - grant/research support, Consultant of: Bayer Eli Lilly - consultant, Speakers bureau: AbbVie, Mustafa Nevzat, Novartis, UCB speaker, Alfred Mahr Consultant of: Celgene, Speakers bureau: Roche, Chugai, Mitsuhiro Takeno Speakers bureau: Esai, Tanabe-Mitsubishi - speaker; Celgene Corporation - advisory board, Doyoung Kim: None declared, Melike Melikoglu: None declared, Sue Cheng Employee of: Amgen Inc. - employment; Celgene Corporation - employment at the time of study conduct, Shannon McCue Employee of: Amgen Inc. - employment; Celgene Corporation - employment at the time of study conduct, Sven Richter Employee of: Amgen Inc. - employment; Celgene Corporation - employment at the time of study conduct, Michele Brunori Employee of: Amgen Inc. - employment; Celgene Corporation - employment at the time of study conduct, Maria Paris Employee of: Amgen Inc. - employment; Celgene Corporation - employment at the time of study conduct, Mindy Chen Employee of: Amgen Inc. - employment; Celgene Corporation - employment at the time of the conduct, Yusuf Yazici Consultant of: BMS, Celgene Corporation, Genentech, Sanofi - consultant, Consultant of: BMS, Celgene Corporation, Genentech, Sanofi - consultant DOI: 10.1136/annrheumdis-2020-eular.2908

\section{OP0029 MAINTENANCE TREATMENT FOR ANCA-ASSOCIATED VASCULITIS IN REAL WORLD PRACTICE IN EUROPE - REALITY OF VASCULITIS REMISSION AND RELAPSE AND SIGNIFICANT BURDEN OF DISEASE}

\section{P. Rutherford'1 , D. Götte'. 'Vifor Pharma, Medical Affairs, Zurich, Switzerland}

Background: After successful remission induction, ANCA associated vasculitis (AAV) is a relapsing remitting long term condition and patients are at risk of organ damage from both active AAV and therapy in particular glucocorticoids (GC). The remission maintenance phase of $\mathrm{AAV}$ is critical for preventing relapse and ensuring organ protection. Objectives: This retrospective study aimed to examine the definition of maintenance start, therapy used and clinical outcomes in patients managed in routine clinical practice. Methods: 1478 AAV patients (France, Germany, Italy, Spain and UK) managed by 493 physicians (37\% Rheumatologists) who completed induction therapy for organ or life threatening AAV and initiated maintenance therapy between 201416 were studied. Data were collected at the time maintenance was determined to begin by the physician and then at $6,12,18$ and 36 months.

Results: $49 \%$ had granulomatosis with polyangiitis,; mean age 54.2 years with $56 \%$ male. $49 \%$ had incident AAV and $51 \%$ were studied from a relapse. $70 \%$ received cyclophosphamide and GC and $30 \%$ received rituximab and GC. Physicians defined time of the start of maintenance from induction treatment start with mean of 5.7 months on basis of fixed time point $40 \%$, starting new drug for maintenance $26 \%$, reaching full remission $26 \%$ and no specific criteria $8 \%$. At this time $43 \%$ were in full remission vs $50 \%$ in partial and $7 \%$ refractory. Various maintenance regimes were used, $21 \%$ received rituximab (88\% 6 monthly and $8 \% 12$ monthly, $4 \%$ other) at varying planned doses $34 \% 1 \mathrm{~g}, 40 \% 500 \mathrm{mg}$ and $23 \% 375 \mathrm{mg} / \mathrm{m} 2,4 \%$ other regime. Remission rates varied, relapse of different severity still occurs and many patients experienced adverse events $(A E)$ and infections with prolonged $G C$ use being common.

At the most recent clinic review patients had been followed for a mean of 50.7 months $-6 \%$ had died, $38 \%$ had relapsed at least once, and $11 \%$ required renal replacement therapy. $54 \%$ had no vasculitis activity, $26 \%$ were ANCA positive

\begin{tabular}{lccccc}
\hline & $\begin{array}{c}\text { Maintenance } \\
\text { start }\end{array}$ & 6 months & 12 months & 18 months & 36 months \\
\hline Remission full/partial \% & $43 / 50$ & $59 / 38$ & $67 / 30$ & $72 / 25$ & $74 / 22$ \\
Total relapse / major \% & 65 & $12 / 49$ & $9 / 45$ & $6 / 44$ & $7 / 32$ \\
Receiving GC\% & 66 & 52 & 53 & 46 & 39 \\
At least one AE \% & 54 & 42 & 32 & 43 & 42 \\
At least one infection \% & & & & & \\
\hline
\end{tabular}

